Macedonian Pharmaceutical Bulletin, 66 (Suppl 1) 179 - 180 (2020)

Online ISSN 1857 - 8969

UDC: $616.311 .2-003.2: 616.314-089.23$

DOI: 10.33320/maced.pharm.bull.2020.66.03.089

Short communication

\title{
Evaluation of biomarker activity in gingival crevicular fluid during short-term orthodontic treatment: comparison between mesial and distal sites
}

\author{
Angela Tasevska ${ }^{1}{ }^{*}$, Liljana Anastasova ${ }^{2}$, Rumenka Petkovska $^{2}$, Natasa Toseska-Spasova $^{1}$, \\ Mirjana Popovska ${ }^{3}$ \\ ${ }^{I}$ Department of Orthodontics, Faculty of Dentistry, Ss. Cyril and Methodius University, \\ 1000 Skopje, Republic of North Macedonia \\ ${ }^{2}$ Institute of Applied Chemistry and Pharmaceutical Analysis, Faculty of Pharmacy, \\ Ss. Cyril and Methodius University, 1000 Skopje, Republic of North Macedonia \\ ${ }^{3}$ Department of Oral Pathology and Periodontology, Faculty of Dentistry, Ss. Cyril and Methodius University, \\ 1000 Skopje, Republic of North Macedonia
}

\section{Introduction}

During orthodontic tooth movement, periodontal tissues respond rapidly to mechanical stress with consequent metabolic changes that allow tooth movement. This process triggers a cascade biological process involving acute inflammatory response in periodontal ligament and consequently alveolar bone remodeling (Dudic et al., 2006; Kumar et al., 2019). The extent of changes during tooth movement can be monitored by performing analysis of biomarkers in gingival crevicular fluid (GCF), such as the enzymes alkaline phosphatase (ALP), lactate dehydrogenase (LDH) and aspartate aminotransferase (AST) (Kapoor et al., 2019; Kumar et al., 2019; Perinetti et al., 2002; Perinetti et al., 2003; Serra et al., 2003). The aim of our study was to assess GCF activity of ALP, AST and LDH from mesial and distal sides of the teeth during the short-term orthodontic treatment. Comparisons between distal and mesial sites were made to evaluate whether application of orthodontic force causes differences in the activity of the above mentioned biomarkers in GCF.

\footnotetext{
*ane_tasevska93@yahoo.com

\section{Materials and methods}

Twenty orthodontic patients (aged 13-28) enrolled in the study underwent bilateral extraction of the first maxillary premolars as part of the orthodontic therapy. Distalization force using elastic chain was applied on one of the maxillary canines representing the test canine (TC) whereas contralateral canine was affected only by the passive force from the stainless steel wire and represented the control canine (CC). Informed consent was obtained from all patients and study protocol was approved by the Ethics Committee of the Faculty of Dentistry. GCF samples were collected before and 7, 14 and 21 days after application of orthodontic force using paper strips (Whatmann, $2 \times 5 \mathrm{~mm}$ ) from the distal (compression) and the mesial (tension) sides of both canines using the method proposed by Koss et al. (2009). After collection, they were placed in eppendorf tubes, $250 \mu \mathrm{L}$ of PBS $\mathrm{pH}=7.4$ (phosphate buffer saline) were added and kept at $-20^{\circ} \mathrm{C}$ until analysis. ALP, AST and LDH activity was determined using commercially available enzyme kits, according to manufacturer's instructions. The statistical analysis was performed using SPSS 20 for 
Windows (SPSS, Chicago, IL, USA). The Wilcoxon signed rank test was employed to evaluate significant differences (at 5\% level $(P \leq 0.05)$ ) between mesial and distal sites, at each time point. Mann Whitney $U$ test was used for independent sample analysis.

\section{Results and discussion}

ALP, AST and LDH activity in GCF measured at the $\mathrm{CC}$ showed no significant changes during treatment as a result of lack of orthodontic force. The low enzyme activity observed in every sampling point shows insignificant bone resorbtion and bone deposition in both sites of CC. Before application of orthodontic force, there were no significant differences in the ALP, AST and LDH activity between the distal and mesial measurement sites of the TC. The mean values for ALP activity on the mesial sites were lower the ALP activity on the distal sites. Significant differences in the mean ALP activity were observed at all occasions after the application of orthodontic force when compared to baseline. Thus, statistically significant differences in GCF ALP activity were seen from day 7 until the end of the treatment with peak enzyme activity measured 14 days after orthodontic force application. However, the comparison of ALP activity between distal and mesial sites revealed no significant differences. These findings indicate that there is an evident ongoing process of bone deposition on both mesial and distal sites of the distalized canine. Mean values for AST activity in GCF were slightly higher on the distal side compared to the mesial side, but the differences were not statistically significant. Peak AST activity was determined on 21 day after beginning of the treatment. This is probably due to the greater bone resorption process that occurs on the compression site. Peak LDH activity was observed on day 21 of the treatment. The analysis of $\mathrm{LDH}$ activity revealed higher enzyme activity on the mesial than the distal site of the tested canine. This finding implies on greater bone resorption that occurs on the compression site of the distalized canine due to distalization force. The comparison of AST and LDH activity on the compression sites revealed no significant differences during short-term orthodontic treatment.

\section{Conclusion}

The application of orthodontic force resulted in changes in the activity of ALP, AST and LDH in GCF. The results suggest that these biomarkers may reflect a part of the biological activity in the periodontium during early phases of orthodontic treatment and therefore be used to prevent and stop adverse effects from the orthodontic treatment.

\section{References}

Dudic, A., Kiliaridis, S., Mombelli, A., Giannopoulou, C., 2006. Composition changes in gingival crevicular fluid during orthodontic tooth movement: Comparisons between tension and compression sides. Eur. J. Oral Sci. 114, 416-422.

Kapoor, P., Monga, N., Kharbanda, O.P., Kapila, S., Miglani, R., Moganty, R., 2019. Effect of orthodontic forces on levels of enzymes in gingival crevicular fluid (GCF): A systematic review. Dent. Press J. Orthod. 24, e1-22.

Koss, M., Castro, S., Lopez, M., 2009. Enzymatic profile of gingival crevicular fluid in association with periodontal status. Lab. Med. 40, 277- 280.

Kumar, G., Rawat, G., Amrita, Kumar, V., Saimbi, C.S., 2019. Evaluation of enzyme activity and rate of tooth movement in corticotomy-accelerated tooth movement - A randomized clinical trial. J. Orthod. Sci. 8, 1-13.

Perinetti, G., Paolantonio, M., Attilio, M.D., Archivio, D., Tripodi, D., 2002. Alkaline phosphatase activity in gingival crevicular fluid during human orthodontic tooth movement. Am. J. Orthod. Dentofac. Orthop. $122,548-556$

Perinetti, G., Paolantonio, M., Attilio, M.D., Archivio, D., Dolci, M., Femminella, B., Festa, F., Spoto, G., 2003. Aspartate aminotransferase activity in gingival crevicular fluid during orthodontic treatment. A short-term longitudinal study. J. Periodontol. 74, 145152.

Serra, E., Perinetti, G., Attilio, M.D., Cordella, C., Paolantonio, M., Festa, F., Spoto, G., 2003. Lactate dehydrogenase activity in gingival crevicular fluid during orthodontic treatment. Am. J. Orthod. Dentofac. Orthop. 124, 206-211. 